\title{
Terapia Respiratoria en Costa Rica: historia y evolución
}

\author{
Respiratory Therapy in Costa Rica: history and evolution
}

Mitzy Vado Chacón ${ }^{1}$ Pilar Chanto Corrales ${ }^{2} \square$

Filiación:

1.Terapeuta respiratorio. Directora de la carrera de Terapia Respiratoria de la Universidad Santa Paula, San José, Costa Rica

Correspondencia: $\square$ Mitzy Vado Chacón

mvado@uspsantapaula.com

2. Terapeuta Respiratorio. Profesora Asistente de la carrera de Terapia Respiratoria de la Universidad Santa Paula, San José, Costa Rica

Correspondencia: $\square$ Pilar Chanto Corrales pchanto@uspsantapaula.com

Forma de citar: Vado Chacón M, Chanto Corrales P. Terapia Respiratoria en Costa Rica: historia y evolución. Rev Ter. 2018;12(1):25-31.

Financiamiento:

Autofinanciado

Conflictos de Interés:

El autor declara no tener conflictos de interés en la presente publicación

\section{Resumen}

La Terapia Respiratoria a través del tiempo ha logrado un rol muy importante en el concepto de salud y se ha expandido en múltiples áreas del cuidado cardiorrespiratorio de la población. Este artículo describe la historia y la evolución del cuidado respiratorio, aportes científicos que permitieron el desarrollo de esta disciplina, y su evolución en Costa Rica, así como las bases que fundamentan el quehacer y los alcances de esta profesión en la actualidad.

Palabras Claves: Terapia Respiratoria, Enfermedades respiratorias, Costa Rica (Fuente: DeCS-BIREME)

\section{Abstract}

Respiratory Therapy through time has achieved a very important role in the concept of health and has expanded into multiple areas of cardiorespiratory care of the population. This article describes the history and evolution of respiratory care, scientific contributions that allowed the development of this discipline, and its evolution in Costa Rica, as well as the bases that support the task and the scope of this profession at present.

Keywords: Respiratory Therapy, Respiratory Tract Diseases, Costa Rica (Source: MeSH-NLM)

\section{Introducción}

La Terapia Respiratoria es una profesión de las ciencias de la salud, que bajo la dirección médica se especializa en la promoción, prevención, diagnóstico, tratamiento y rehabilitación de personas con enfermedades agudas o crónicas que afectan directa o indirectamente el sistema cardiorrespiratorio. Es una disciplina ejercida por Terapeutas Respiratorios, sin embargo, según el país del que se esté haciendo referencia también estos profesionales son llamados kinesiólogos, fisioterapeutas respiratorios o especialistas cardiopulmonares.

Un gran porcentaje de estos profesionales trabajan en hospitales, otros en clínicas 
de mediana complejidad, laboratorios de función pulmonar, clínicas de sueño, clínicas estéticas, centros de rehabilitación cardiopulmonar y hogares destinados al cuidado de personas adultas mayores o con discapacidad. Algunos otros Terapeutas respiratorios trabajan en domicilio, en el área prehospitalaria, en industrias en el área de salud ocupacional, en empresas como asesores clínicos y otros en instituciones educativas en la formación de estudiantes o de otros profesionales afines.

\section{Antecedentes históricos}

Los conceptos asociados a ciencia y salud se remontan desde hace muchos años y siguen avanzando hasta la actualidad.

La Sagrada Biblia hace mención de un concepto relacionado al cuidado respiratorio, cuando en su primer libro Génesis dice: "Entonces Jehová Dios formó al hombre del polvo de la tierra, y sopló en su nariz aliento de vida, y fue el hombre un ser viviente"1, haciendo referencia a la respiración como un signo vital.

En el periodo arcaico Hipócrates médico de la antigua Grecia "Padre de la medicina" basó sus conceptos en cuatro fluidos esenciales flemas, sangre, bilis amarilla y bilis negra y en cuatro elementos tierra, fuego, agua y aire $^{2}$, términos contemplados en el cuidado cardiorrespiratorio.

Como dice Wilkins y colaboradores ${ }^{3}$, durante el periodo contemplado entre el siglo XVII y $X X$, aparecen grandes aportes científicos que fundamentan la fisiología respiratoria, la patología y el cuidado cardiopulmonar. (Ver Cuadro 1)

Según Varela ${ }^{4}$, específicamente en Costa Rica la peor epidemia de poliomielitis que se registro fue en 1954 con 1.081 casos de niños con discapacidades y más de cien muertos. Durante ese tiempo, las personas afectados por la enfermedad fueron atendidos, en el Hospital San Juan de Dios, único centro hospitalario de la capital, donde lo primordial era salvarles la vida, pues muchos menores llegaban con serios problemas respiratorios y su muerte podía sobrevenir en cualquier momento, situación donde que requirió de la utilización de los "pulmones de acero", ventiladores mecánicos de presión negativa que según Salas Segura5, gracias a los esfuerzos del Dr. Humberto Araya Rojas pediatra y a una asociación de Damas Voluntarias se trajeron dos pulmones desde México al Hospital San Juan de Dios, uno pediátrico y uno de adultos, donde este último siguió utilizándose en pacientes con intoxicación por insecticidas y que cerca de la década de los setentas fueron sustituidos por ventiladores de presión positiva.

Sin embargo ahí la historia no acaba los avances médicos permitieron la prevención de enfermedades a través de las vacunas, el diagnóstico de enfermedades o problemas cardiopulmonares a través del estudio con rayos $\mathrm{X}$ cuyo descubrimiento en 1901 cambio el ritmo de los diagnósticos16, así como la toma y análisis de gases arteriales, las mediciones de volúmenes y capacidades pulmonares con el espirómetro, el oxímetro de oído y posteriormente de pulso y la polisomnografía son otros de los procedimientos que siguen permitiendo que los terapeutas respiratorios apoyen el diagnostico medico de una persona enferma o que den seguimiento de la intervención aplicada.

\section{El avance de la profesión}

Los primeros terapeutas respiratorios en Norteamérica eran los llamados "técnicos en oxígeno", y se encargaban de movilizar los cilindros y colocar los dispositivos para la oxigenoterapia, como las mascarillas, tiendas y cánulas nasales, sin embargo la profesión fue creciendo cuando se descubrieron más y mejores dispositivos para la entrega de ese gas y se crearon técnicas para 


\section{Cuadro 1. Eventos históricos asociados a la Terapia Respiratoria .}

\begin{tabular}{|c|c|c|}
\hline Año & Personaje & Evento histórico \\
\hline 1643 & Evangelista Torricelli & $\begin{array}{l}\text { Invento el primer barómetro del mundo para medir la } \\
\text { presión atmosférica. }{ }^{7}\end{array}$ \\
\hline 1662 & Robert Boyle & $\begin{array}{l}\text { Describe la relación entre la presión y el volumen de } \\
\text { los gases }^{8}\end{array}$ \\
\hline 1738 & Daniel Bernoulli & $\begin{array}{l}\text { Determina que conforme la velocidad de un líquido } \\
\text { aumenta la presión disminuye. } .^{8}\end{array}$ \\
\hline 1774 & Joseph Priestley & Describió su descubrimiento del oxígeno ${ }^{7}$ \\
\hline 1787 & Jacques Alexander Cesar Charles & $\begin{array}{l}\text { Describe la relación entre el volumen y la tempera- } \\
\text { tura }^{7}\end{array}$ \\
\hline 1800 & William Henry & $\begin{array}{l}\text { Determina que la cantidad de gas disuelta en un } \\
\text { líquido es directamente proporcional a la presión } \\
\text { parcial }^{9}\end{array}$ \\
\hline 1800 & Adolph Fick & $\begin{array}{l}\text { Descubre un método para calcular gasto cardiaco } \\
\text { con consumo de oxígeno y contenido arterio-venoso } \\
\text { de oxígeno. }{ }^{9}\end{array}$ \\
\hline 1801 & John Dalton & $\begin{array}{l}\text { Describe la relación de las presiones parciales en } \\
\text { una mezcla de gases. }\end{array}$ \\
\hline 1806 & Simon de la Place & $\begin{array}{l}\text { Describe la relación entre la presión y la tensión su- } \\
\text { perficial en gotas de líquido }{ }^{8}\end{array}$ \\
\hline 1808 & Joseph Louis Gay Lussac & $\begin{array}{l}\text { Describe la relación entre la presión y la Temperatu- } \\
\mathrm{ra}^{7}\end{array}$ \\
\hline 1831 & Thomas Graham & Describe la difusión de dos gases ${ }^{9}$ \\
\hline 1846 & John Hutchinson & Desarrolla el espirómetro ${ }^{10}$ \\
\hline 1864 & Alfred F. Jones & $\begin{array}{l}\text { Patenta un equipo de presion negativa para soporte } \\
\text { ventilatorio }{ }^{11}\end{array}$ \\
\hline 1895 & William Roentgen & Descubre los Rayos X ${ }^{12}$ \\
\hline 1909 & Samuel James Melltzer & Introduce la Intubación orotraqueal ${ }^{11}$ \\
\hline 1911 & Heinrich Drager & $\begin{array}{l}\text { Desarrolla el ventilador mecánico para el uso en re- } \\
\text { animación }{ }^{11}\end{array}$ \\
\hline 1913 & Chevalier Jackson & $\begin{array}{l}\text { Desarrolla un laringoscopio para la inserción de tu- } \\
\text { bos endotraqueales }{ }^{12}\end{array}$ \\
\hline 1928 & Phillip Drinker & $\begin{array}{l}\text { Desarrolla un respirador con presión negativa "pul- } \\
\text { món de acero"11 }\end{array}$ \\
\hline 1948 & Bennett & Lanza un ventilador de presión positiva ${ }^{13}$ \\
\hline 1958 & Bird & Lanza un ventilador de presión positiva ${ }^{13}$ \\
\hline 1970 & Bird & Se crea el primer ventilador neonatal ${ }^{14}$ \\
\hline 1972 & El ventilador Siemens fue lanzado ${ }^{13}$ & \\
\hline
\end{tabular}

Fuente: Elaboración de los autores 
mejorar otras condiciones como lo fue la aerosol terapia, asociada a la implementación de fármacos inhalados, la inserción y cuidado de las vías aéreas tanto anatómicas como artificiales ${ }^{15}$, las técnicas de higiene broncopulmonar, las técnicas de expansión y por supuesto la ventilación mecánica.

El momento crítico en la historia de la humanidad que dio auge a lo que actualmente es una de las principales funciones de los terapeutas respiratorios, fue la epidemia del polio que se mencionó anteriormente. En 1928 Drinker había desarrollado su pulmón de hierro y Emerson en 1930 y fue en estas epidemias de 1940 y 1950 donde se introdujo la utilización de los ventiladores mecánicos de presión negativa y algunos años más tarde los ventiladores de presión positiva de Drager, Bird y Bennett. Desde entonces se han introducido más de 50 ventiladores nuevos con diversas características para el uso clínico, pero con la misma esencia de los antiguos

Según Wilkins ${ }^{3}$, el primer curso de educación formal en terapia de inhalación se ofreció en Chicago, Estados Unidos en 1950, y para la década de 1960 se desarrollaron numerosas escuelas para entrenar a los terapeutas respiratorios. Los primeros programas se concentraron en enseñar a los estudiantes la aplicación adecuada de la oxigenoterapia, aerosolterapia, y dispositivos de ventilación con presión positiva intermitente (IPPB). Ya posteriormente en los años 60 y 70 la ventilación mecánica para pacientes críticos y dispositivos para monitoreo permitieron impulsar más el papel del terapeuta respiratorio como experto cardiopulmonar.

En Costa Rica, la terapia respiratoria inicio formalmente a mediados de la década de los setenta donde se inician las Unidades de Cuidado Intensivo en los principales hospitales de la Caja Costarricense del Seguro Social (CCSS) en el área metropolitana. Los jefes médicos de estas unidades se especializaron en medicina crítica y cuidado intensivo en Estados Unidos y Canadá, allá conocieron el desempeño del Terapeuta Respiratorio y a su regreso a nuestro país propiciaron, de diferentes formas, la creación de un programa de formación de estos recursos humanos, esfuerzos liderados por el Dr. Guillermo Rodríguez Gómez y que culminaron con un convenio interinstitucional entre la Universidad de Costa Rica (UCR), CCSS y la Fundación Norteamericana Proyecto HOPE (People to People Health Foundation Inc.), esta última aportó el personal docente y equipos necesarios para iniciar la formación de Terapeutas Respiratorios en nuestro país con proyección centroamericana.

La formación de Terapeutas Respiratorios en Costa Rica se inició en 1985, a través de la Escuela de Enfermería de la UCR como parte del convenio tripartita entre las instituciones anteriormente mencionadas, avalado por el Gobierno de Costa Rica que se publicó en el diario oficial La Gaceta No 176, del 17 de septiembre de 1983. El primer programa se concibió dentro del concepto de formación académica superior universitario, llamado "Programa de Especialización en Terapia Respiratoria” y estuvo adscrito a la Vicerrectoría de Docencia de la UCR. EI segundo programa se concibió como un Programa de Capacitación adscrito a la Vicerrectoría de Acción Social de la misma Universidad. Ambos programas tuvieron una proyección a nivel centroamericano. Estos programas tuvieron una duración de un año lectivo cada uno, y estaban dirigidos a enfermeras con experiencia en cuidados intensivos. De ellas se graduaron treinta y tres estudiantes, de las cuales, trece eran extranjeras. Posteriormente, en 1986, la formación de estos recursos humanos paso de la Escuela de Enfermería a la Escuela de Medicina de la UCR, y dentro de la Escuela de Medicina se ubicó en el Departamento de Tecnologías en Salud.

Durante el periodo 1987-1989 se ofrece, por una sola vez, el plan de técnico en Terapia Respiratoria, que lograron concluir siete estudiantes, quienes se 
incorporaron posteriormente en el diplomado, que se ofreció desde 1988. En 1991 se aprueba la carrera de Bachillerato en Terapia Respiratoria para ser impartida en este mismo departamento. Esta carrera tenía una duración de cuatro años y se ofreció en forma paralela al diplomado, no conformando una sola carrera e impedir de esta forma a los estudiantes graduados del diplomado continuar con el bachillerato. De este último solamente se abrió una promoción, finalmente el programa de formación de Terapeutas Respiratorios de la UCR cerró en julio de 1995.

Considerando que la Terapia Respiratoria es una profesión con conocimientos especializados, habilidades y destrezas que van más allá de la ejecución de acciones simples. Además, los principios científicos, el análisis y la interrelación de datos permiten reconocer que el Terapeuta Respiratorio no podía seguir siendo un técnico, el rol de este personal de salud es un rol profesional, donde no solo se siguen las órdenes de un médico, sino más bien evalúa al paciente y en base a esto desarrolla un plan de intervención terapéutica y vuelve a evaluar para ajustar su intervención a las necesidades integrales de esa persona. Las áreas de desempeño del profesional en terapia respiratoria tienen mayor alcance y van más allá de simplemente ajustar parámetros de un ventilador. Bajo esta perspectiva, en el año 1998, la Universidad Santa Paula, a través de su fundadora y rectora, la Licda. Rocio Valverde Gallegos, apertura los niveles de bachillerato y licenciatura en Terapia Respiratoria, formando profesionales de manera ininterrumpida hasta la actualidad.

El Programa de Terapia Respiratoria de la Universidad Santa Paula (USP), se ha mantenido pionero y líder en esta área, consolidándose como el único programa a nivel nacional, y obteniendo en el año 2017 la Acreditación de la carrera por parte del Sistema Nacional de Acreditación de la Educación Superior (SINAES), posterior a un riguroso proceso de evaluación interna y externa con pares evaluadores de Chile y Estados Unidos. Durante el periodo 2002-2017 se han graduado 422 licenciados en terapia respiratoria, los cuales laboran en el territorio nacional y en el extranjero (Figura 1), y pese a ser un programa relativamente joven, los licenciados en terapia respiratoria destacan en áreas de alta responsabilidad como son las unidades de cuidados intensivos ${ }^{14}$. Además, desde la USP este programa es sometido a constantes procesos de mejora, tanto en sus planes de estudio y buenas prácticas en los procesos de enseñanza aprendizaje, educación continua a través de cursos libres, cursos de especialización y programas de maestría, como en infraestructura y tecnología.

Los retos actuales de la terapia respiratoria en nuestro país, así como en otros países del mundo se orienta en participar en áreas como la rehabilitación cardiopulmonar, las clínicas de cesación de fumado, los laboratorios para trastornos del sueño, la apertura de servicios en el primer y segundo nivel de atención ${ }^{12}$, logrando que los pacientes con asma, EPOC y otras enfermedades crónicas puedan prevenir reagudizaciones y poder ingresar en un programa de rehabilitación cardiaca ${ }^{16}$, la apertura de espacios en todos los hogares de ancianos y los centros de personas con discapacidad, en instituciones educativas y la apertura de espacios de asistencia a médicos en el área de anestesiología y neumología. Así como el reforzamiento del trabajo inter y transdiciplinario ${ }^{17}$ y la intervención a través de telemedicina ${ }^{15}$.

La respiración es uno de los signos vitales más importantes para la preservación de la vida, y uno de los elementos que pueden causar mayor desesperación en caso de fallo. La terapia respiratoria sigue en un proceso dinámico de evolución, en un contexto de equipos multi-inter y transdiciplinarios cuya composición va a depender 


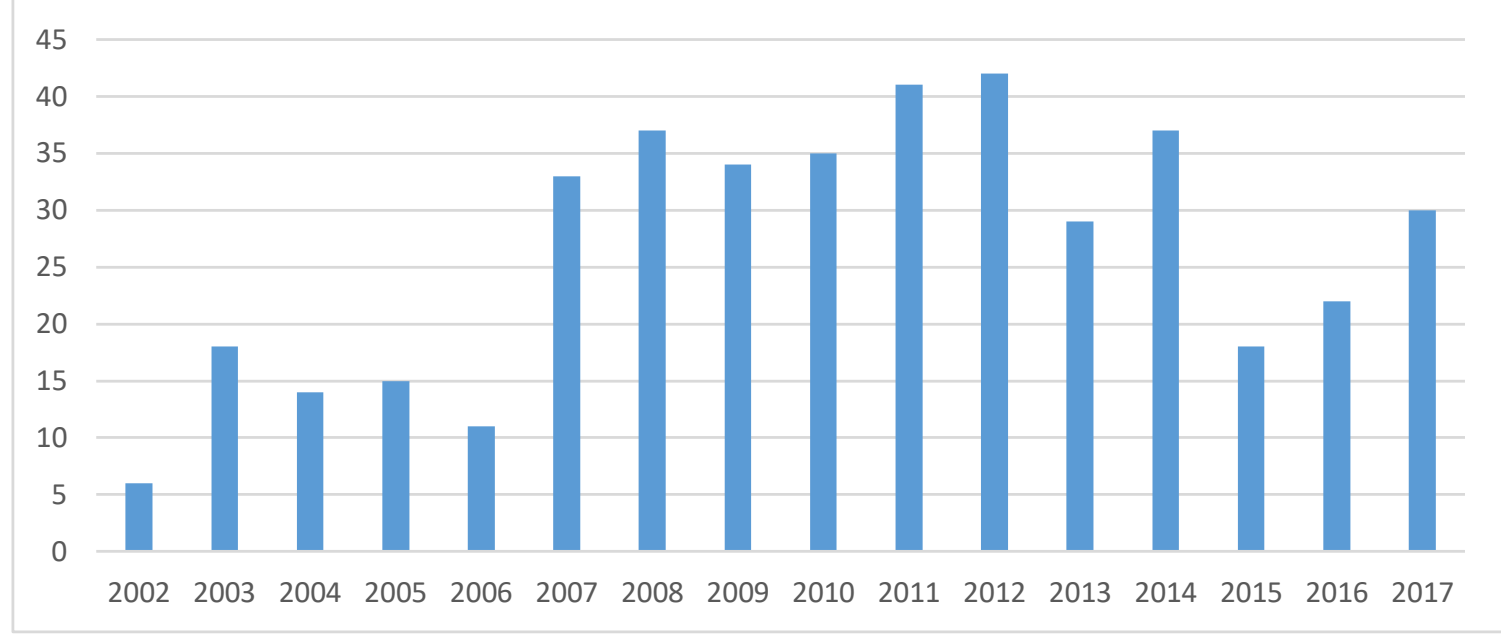

Figura 1. Número de graduados en Terapia Respiratoria, Universidad Santa Paula 2002-2017 Fuente: Departamento de registro. Universidad Santa Paula, 2018.

de las características de su lugar de trabajo, del mismo terapeuta respiratorio y de las distintas áreas y servicios privados y públicos de salud.

\section{Referencias Bibliográficas}

1. La Sagrada Biblia. Versión Reina Valera 1960: Sociedades Bíblicas Unidas; 1988. Genesis 2:7.

2. Bounfante Martínez M. La influencia del estado anímico del paciente oncológico en relación con el resultado de la quimioterapia [Tesis en Enfermería]. Mallorca: Universidad de las Islas Baleares; 2017.

3. Wilkins R, Stoller J, Kacmarek R. Egans Fundamentals of Respiratory Care. 10a ed. Missouri: Elsevier; 2013.

4. Varela I. Para que nunca se repita... [Internet]. La Nación. 2002 [citado el 04 de abril del 2018]. Disponible en http://wvw.nacion.com/ dominical/2002/marzo/17/dominical0.html

5. Salas Segura D. Breve historia de la venti- lación mecánica asistida. Acta Acad. 2000; 89-91.

6. Dávalos Villca MV. Historia de la radiología. Rev Act Clin Med [Internet]. 2013;37: 17871792. Disponible en: http://www.revistasbolivianas.org.bo/pdf/raci/v37/v37a01.pdf

7. Cairo JM. Respiratory care equipment. 10a ed. Missouri: Elsevier; 2018.

8. Cristancho W. Fisiología respiratoria. Lo esencial en la práctica clínica. 3a ed. Colombia: Manual Moderno; 2012.

9. West J, Luks A. Fisiología respiratoria. Fundamentos. 10a ed. Barcelona: Wolters Kluwer; 2016.

10. Mottram C. Ruppels manual of pulmonary function testing. 11a ed. Missouri: Elsevier; 2018.

11. Cristancho W. Fundamentos de fisoterapia respiratoria y ventilación mecánica. 3a ed. Bogotá: Manual Moderno; 2015. 
12. Smith SG, Endee LM, Benz Scott LA, Linden PL. The Future of Respiratory Care: Results of a New York State Survey of Respiratory Therapists. Respir Care. 2017;62(3):279287.

13. Oakes D, Shortall S, Jones S. Ventilator management. 4a ed. Clayton: Health Educator Publications; 2016.

14. Roxby P. The flying fanatic who helped babies breathe [Internet]. BBC News Services. 11 de octubre de 2015. Disponible en: http:// www.bbc.com/news/health-34482260

15. Tobin M. Principles and practice of mechanical ventilation. 3a ed. McGraw Hill; 2013.

16. Betancourt Peña J, Mosquera García AC, Orozoco Henao LM, Parra Lievano JA, Velasco Rivera E, Ávila Valencia JC, et al. Rehabilitación pulmonar: Caracterización de pacientes con enfermedad pulmonar obstructiva crónica que inician un programa de rehabilitación pulmonar. Mov Cient. 2018;11(2):4754.

17. Zamjahn JB, Beyer EO, Alig KL, Mercante $\mathrm{DE}$, Carter KL, Gunaldo TP. Increasing Awareness of the Roles, Knowledge, and Skills of Respiratory Therapists Through an Interprofessional Education Experience. Respir Care. 2018;63(5):510-518.

18. American Association for Respiratory Care [Internet]. Texas: AARC; 2015 [20 abril 2017]. PACT day efforts promote telehealth bill. Disponible en: http://www.aarc.org/pact-day-efforts-promote-telehealth-bill/. 